\title{
Membrane Lipid Switches: How Membrane Lipid Structure Influences Protein-Lipid Interactions
}

\author{
Created by: Manuel Torres, QCatalina Ana Rosselló , QPaula Fernández-garcía, \\ QVictoria Llado, QPablo V Escribá \\ Revised by: Pablo V Escribá \\ Version received: 23 April 2020
}

check for

updates

\begin{abstract}
Peripheral membrane proteins are required for signal propagation upon ligand-induced receptor activation at the plasma membrane. The translocation of this amphitropic peripheral proteins from or to the plasma membrane enables signal cascade propagation into the cells. This translocation greatly depends on the membrane's lipid composition and, consequently, regulation of the lipid bilayer emerges as a novel therapeutic strategy. Indeed, relevant changes in membrane lipids can induce massive translocation of peripheral signaling proteins from or to the plasma membrane, which controls how cells behave. We called these changes "lipid switches", as they alter the cell's status (e.g., proliferation, differentiation, death, etc.) in response to the modulation of membrane lipids. This discovery enables therapeutic interventions focused on modifying the bilayer's lipids, an approach known as membrane-lipid therapy (MLT) or melitherapy.
\end{abstract}

\section{Introduction}

Amphitropic membrane proteins are required for signal propagation upon ligand-induced receptor activation at the plasma membrane. These proteins are only activated by ligand-receptor complexes when they both come into physical contact. The interaction between membrane receptors and the amphitropic proteins may not only depend on the expression of these proteins but also on the presence of the peripheral proteins in the vicinity of the membrane receptor, which may be controlled by membrane lipids $[\underline{1}][\underline{2}]$. Therefore, changes in the membrane lipid composition can induce important changes in cell physiology that affect proliferation, differentiation, and/or cell death $[3][4]$. These interactions and the signals they produce are responsible for the pathophysiological status of the cell, which may be influenced by external cues, genetic alterations, lipid storage disorders, etc. $[\underline{5}][\underline{6}]$. Recent studies have shown that the type and levels of peripheral amphitropic signaling proteins in membranes or aqueous compartments depends on both the membrane's lipid composition, and the protein's amino acid sequence and post-translational lipid modifications[ㄹ][]ㅡ. In this sense, alterations in the balance of peripheral signaling proteins at membranes and in the cytosol have been associated with a variety of pathologies $[\underline{[}][\underline{9}]$. The regulation of membrane lipids controls the type and abundance of the proteins in membranes, an approach that can be used to treat several conditions, including cancer, Alzheimer's disease (AD), cardiovascular diseases (CVDs), inflammation, etc. [10][11][12][13].

\section{Influence of Membrane Lipid Structure and Composition on Protein-Lipid Interactions}

This entry aims to review the interaction of amphitropic signaling proteins with membrane structures. This type of interaction deserves further attention because: (i) the plasma membrane is a critical hub for signaling proteins; (ii) cells can regulate their lipid composition according to a range of pathophysiological situations; (iii) membrane lipids organize into different microdomains rich in specific lipid species, which attract different types of proteins; and (iv) proteins that prefer certain types of lipid structures can drive productive interactions involving the reception and propagation of cell signals in

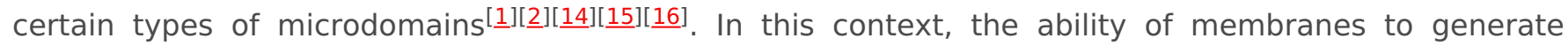
microdomains is due to the non-homogeneous mixing of membrane lipids[1]][18]. One example of this heterogenous lipid mixture is the transbilayer lipid asymmetry. Higher levels of sphingomyelin (SM) and phosphatidylcholine (PC) have been found in the outer plasma membrane leaflet, whereas phosphatidylethanolamine (PE) and phosphatidylserine (PS) are more abundant in the inner leaflet. This asymmetry has a relevant impact on the biophysical properties of the membrane and the protein-lipid 
interactions ${ }^{[19]}$. Indeed, the number of peripheral proteins bound to the inner leaflet is higher than that bound to the outer leaflet ${ }^{[20]}$. A variety of microdomains have been described in which either lamellarprone or non-lamellar-prone lipids organize into different ordered or disordered lipid

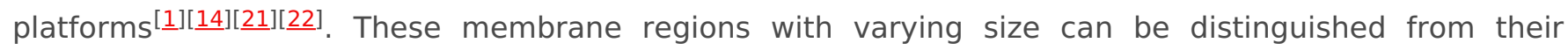
adjacent microdomains in terms of their lipid and protein composition, bilayer thickness, lateral surface pressure, acyl chain mobility, membrane morphology, etc.

In general, the formation of membrane microdomains with specific lipids favors the presence of certain peripheral proteins, while hindering the interaction of other proteins. For example, microdomains with a high proportion of hexagonal $\left(H_{\|}\right)$phase-prone lipids, such as PE or diacylglycerol (DAG), are critical in the recruitment of peripheral amphitropic signaling proteins and thus, for cell growth and differentiation $[\underline{1}][\underline{15}]$. Indeed, the interaction of peripheral membrane proteins, such as $G$ proteins and Protein Kinase C (PKC), with HII membrane structures was first described some years ago[1]. In this context, one of the mechanisms of action by which anthracyclines exert their antitumor action was through the inhibition of HII-phase propensity and the subsequent mislocalization of these signaling proteins. This phenomenon explained why anthracyclines could kill cancer cells solely by interacting with the plasma membrane but not entering the cells $\underline{[23]}$. Subsequently, important modifications of the plasma membrane's lipid composition by anthracyclines was seen to be relevant to their mechanism of action[ [3] .

One example of membrane microdomain in cells are caveolae ("little caves") which form spatiotemporal platforms where Endothelial Growth Factor Receptor (EGFR), Ras, and Raf1 meet to propagate signals promoting cell growth ${ }^{[24]}$. Similarly, in the case of G proteins, Liquid-ordered (Lo) microdomains (e.g., lipid rafts) are preferred by Gail proteins, whereas Liquid-disordered (Ld) microdomains bind with high affinity to $G \alpha \beta$ and $G \alpha \beta \gamma$ proteins $[\underline{2]}[\underline{15}]$. In fact, $G \alpha \beta$ heterodimer was seen to drive the interaction of $\mathrm{G} \alpha \beta$ y heterotrimers in PE-enriched Ld membrane microdomains. Thus, one of the roles of G $\alpha \beta$ dimers is to bring $\mathrm{G} \alpha$ monomers into contact with G-Protein-Coupled Receptors (GPCRs)[ㄹ] $[\underline{15}][\underline{7}]$. Another wellknown protein-membrane interaction is the $\mathrm{Ca}^{2+}$-mediated fusion of synaptic vesicles to membranes in order to release neurotransmitters into the synaptic cleft. In this process, $\mathrm{Ca}^{2+}$ binding to the $\mathrm{C} 2$ domain of synaptotagmin mediates vesicle exocytosis, assisting fusion to the plasma membrane via its interaction with a SNARE/complexin complex in presynaptic terminals $[\underline{25]}$. In general, non-lamellar-prone membrane microdomains rich in PE or DAG are necessary for interactions with the C2 domain in certain proteins. Moreover, they are necessary for membrane fusion and fission phenomena, such as exocytosis and endocytosis, which require the formation of inverted curvature non-lamellar (HII) intermediates $[\underline{26]}[\underline{27]}[\underline{28}]$.

In summary, the localization and activity of important peripheral signaling proteins is very sensitive to changes in membrane structure ${ }^{[1]}$. Therefore, natural or synthetic molecules that regulate lipid polymorphism in vitro and membrane microdomains in vivo ${ }^{[29]}$ can regulate the localization and activity of peripheral membrane proteins, and thereby modulate cell signaling. In this context, membrane microdomains act as sites where signaling partners exert productive interactions. As such, signaling proteins can interact with downstream signal transducers, sharing their affinity for certain membrane lipids or lipid structures. Lamellar-prone Lo membrane microdomains (e.g., lipid rafts) contain specific lipids that define their membrane lipid structure and that are involved in selecting the proteins that bind to them $[\underline{2}][\underline{30]}$. The ability of lipids to organize into different structures (lipid mesomorphism) depends on the lipid composition and external physical factors, such as temperature. The mosaic of lipid structures that defines different membrane microdomains facilitates a number of different protein-lipid interactions $[\underline{14}][\underline{21}][\underline{31]}$.

\section{Altered Protein-Lipid Interactions in Human Disease and Therapy}

The activity of many amphitropic proteins depends on their membrane interactions, which are modulated by the lipid composition of the membrane. The activity of several important signaling proteins is regulated by protein-lipid interactions, including Src kinase, RAS-guanine nucleotide 
exchange factor, cytidylyltransferase, PKC, phospholipase C, vinculin and DnaA protein. Therefore, membrane lipids imbalance can have an important influence on several diseases, as recently reviewed $^{[32]}$ (Figure $\mathbf{1}$ ).

For example, cystic fibrosis causes lipid imbalances that affect surfactant function, producing a negative effect on breathing[33][34]. In mouse models of cystic fibrosis, a similar lipid imbalance was found in affected organs, although administration of docosahexaenoic acid (DHA) normalized both these lipid changes and the animal's health status ${ }^{[35]}$. In brain injury, a significant increase in SM, PE, PC and the derivatives lysoPE and lysoPC have been described at acute and/or sub-acute time points[36]. In diabetes, DAG levels are chronically elevated in various tissues, such as the retina, aorta, heart and renal glomeruli, liver and skeletal muscles, leading to abnormal PKC activation [37]. PKC membrane recruitment is accompanied by a conformational rearrangement that relieves auto-inhibitory interactions, enabling PKC to bind to membranes through its $\mathrm{C} 1$ and/or $\mathrm{C} 2$ domains, and allowing it to phosphorylate its targets[38][39]. On the other hand, sphingolipids appear to be critical in the prognosis of anaplastic lymphoma. Thus, Anaplastic Lymphoma Kinase (ALK)+ lymphomas may express an ALK fusion protein involved in cancer cell survival, or the Cbp/PAG adaptor protein and the Lyn kinase signalosome (a protein complex involved on signal propagation) that recruits other transcription factors and signaling enzymes. Lyn is not particularly active in ALK+ lymphoma membranes that contain sphingolipid-rich domains (i.e.: raft-like membrane microdomains) which impairs the productive signaling of the Lyn-Cbp/PAG signalosome ${ }^{[\underline{40]}}$. Therefore, the plasma membrane appears to act as a switch, and alterations in its composition cause dramatic translocations of proteins to or from the plasma membrane. Such signals appear to be especially relevant in the context of cell proliferation. Thus, either the increase in cell proliferation caused by tumor alterations or decreased proliferation related to neurodegeneration (e.g., AD or Parkinson's disease (PD)) have been related to membrane lipid modifications $[\underline{1}][\underline{3}][\underline{11}][\underline{41}][\underline{42}]$.

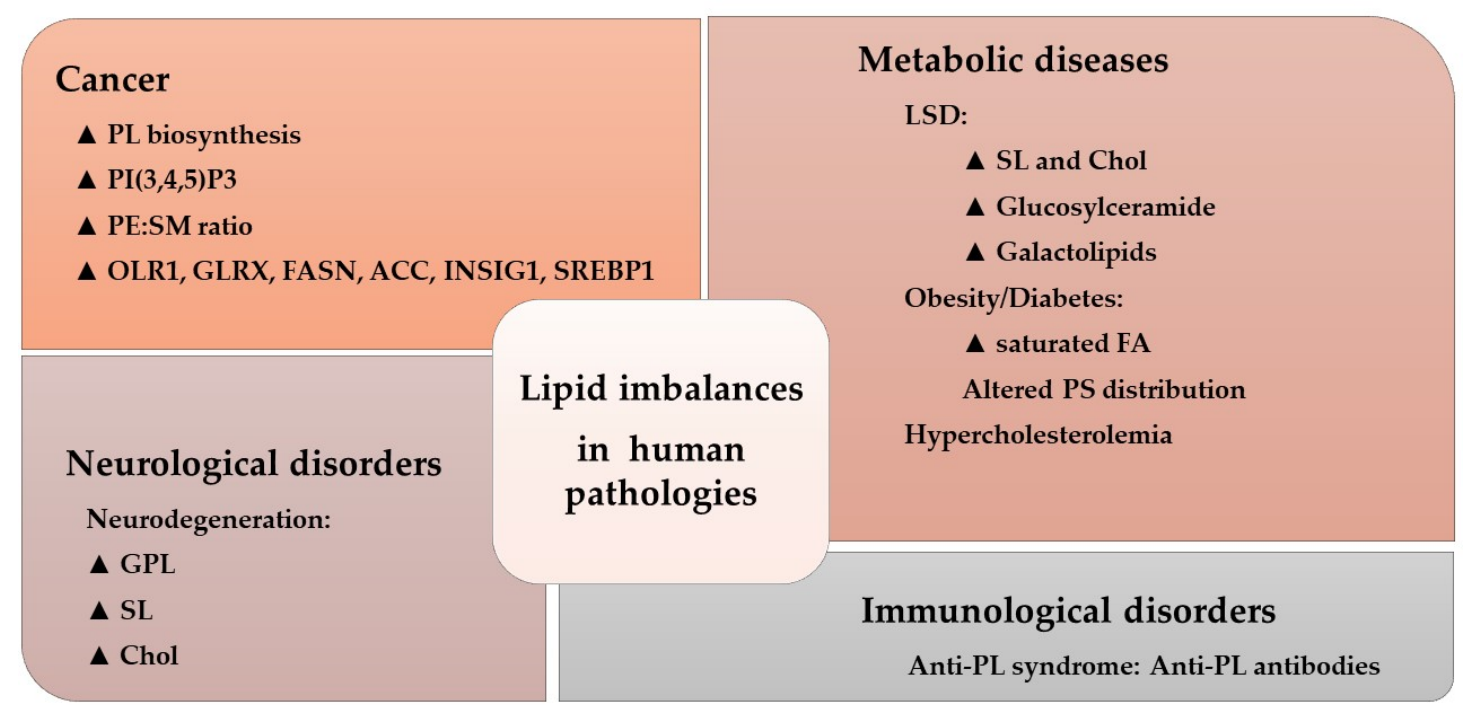

Figure 1. Lipid imbalances and human pathologies. Alterations to the lipidome in a variety of conditions. The triangle indicates increased levels or pathway activity: PL, phospholipid; PtdIns(3,4,5)P3, phosphatidylinositol 3,4,5-trisphosphate; PE, phosphatidylethanolamine; SM, sphingomyelin; OLR1, oxidized low-density lipoprotein receptor 1; GLRX, glutaredoxin; FASN, FA synthase; ACC, acetyl-CoA carboxylase; INSIG1, insulin induced gene 1; SREBP1, sterol regulatory element-binding protein 1; LSD, lysosomal disorder; SL, sphingolipid; Chol, cholesterol; FA, fatty acid; PS, phosphatidylserine (Adapted from [32]).

\section{Protein-Lipid Interactions in Cancer}

The RAS family of amphitropic proteins is related to cell proliferation and mutated (especially K-RAS) in 
$95 \%$ of pancreatic, $45 \%$ of colorectal, and $35 \%$ of lung cancers ${ }^{[43]}$. Guanine nucleotide exchange factors (GEFs) and GTPase activating proteins (GAPs) control RAS activation by inducing GDP exchange for GTP or GTP hydrolysis to GDP, respectively. To regulate RAS activation, GEFs and GAPs are recruited to plasma membrane microdomains close to RAS. The activity of K-RAS has been directly related to membrane regions rich in PS which interact with a polybasic amino acid region in the C-terminal region of this protein [44] . In addition, RAS activation requires palmitoylation at Golgi membranes that drives RAS to the plasma membrane via vesicle trafficking ${ }^{[45]}$. The presence of RAS at the plasma membrane is necessary for its activity as a tumor promoter, which also depends on its covalent acylation. Palmitoylation is not only important for RAS activity, it is also essential for the function of other oncogenes (e.g., EGFR).

The Wnt signaling pathway regulates a variety of cellular processes including cell proliferation. Hence, aberrant activation of the Wnt-FZD signaling leads to tumorigenesis in many tissues ${ }^{[46]}$, including the breast, prostate, colon, brain and pancreas. Wnt family members undergo two types of post-translational modifications that influence their interactions with lipid bilayers and that are essential for Wnt signaling: serine acylation and the subsequent S-palmitoylation of cysteine[4] ${ }^{[47}$. Wht signaling involves crosstalk with other important cell signaling pathways including the Notch, Hedgehog, and EGFR cascades ${ }^{[48]}$ which are all of them altered to some degree in different cancers $[\underline{49}][\underline{50]}[\underline{51]}$ and controlled by lipid-protein interactions, which highlights the relevance of these interactions in cancer. Accordingly, modulation of these lipid-protein interactions may produce potential therapeutic benefits in the

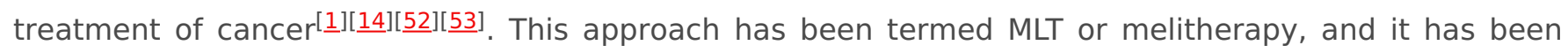
demonstrated to combine high efficacy and safety in clinical trials (e.g., ClinicalTrials.gov identifiers NCT01792310 and NCT03366480).

\section{Protein-Lipid Interactions in Neuroregeneration}

Neurodegenerative diseases are a public-health issue worldwide with unmet clinical needs. Classic therapies focus on preventing or delaying neuronal degeneration, whereas more recent interest has also focused on neuroregenerative therapies. The finding that Neural Stem/Progenitor Cells (NSPCs) persist in adults, and the discovery of relevant transcription factors and signaling pathways, including signaling lipids that influence NSPC behavior and of neurogenesis, raised hope in therapies based on NSPC regulation and the potentiation of neurogenesis ${ }^{[54]}$. In this context, polyunsaturated fatty acids (PUFAs), like DHA (C22:6, n-3) and AA (arachidonic acid, C20:4, n-6) are abundant in the CNS, being the brain the organ with the highest DHA levels $[\underline{55]}$. Studies reviewing the effect of these PUFAs on NSPC regulation support a role for both in neurogenesis during brain development and adulthood. Specifically, AA increases NSPC proliferation, and it probably influences the maintenance of the NSPC pool, whereas DHA promotes neuronal differentiation[ [56][그. In addition, not only do the individual levels of these two PUFAs in cell membranes play a role in neurogenesis but also, the ratio between them is determinant as a lipid switch ${ }^{[58]}$. In fact, due to the higher proportion of omega- 6 PUFAs in western diets, low dietary omega6/omega-3 ratios have been widely described as beneficial on neurodegenerative pathologies such as AD.

PUFAs can modulate lipid-raft-mediated signaling by regulating the composition of these structures[리 $[\underline{59]}$. For instance, increased levels of cell membrane PUFAs are associated with increased NSPC proliferation due to the disruption of protein localization to lipid rafts $[\underline{60]}$. Membrane lipids can also regulate signaling in NSPCs through fatty acid (FA) binding to specific receptors, such as Fatty Acid Binding Proteins (FABPS). Three members of this family are expressed in the brain: FABP3, FABP5 and FABP7 [1] . The protein FABP3, is related with neuritogenesis and synaptogenesis, whereas FABPs 5 and 7 are involved in NSPC differentiation and migration ${ }^{[62]}$. Other receptors influenced by DHA and other PUFAs and that are involved in neurogenesis have also been described. Thus, DHA has been shown to bind (directly or via FABPs) to Peroxisome Proliferator-Activated Receptor Y (PPARY), a nuclear receptor 
that mediates the expression of transcription factors that enhance neurogenesis ${ }^{[63]}$. DHA also binds to GPR40 (G-protein coupled receptor 40), the activation of which leads to neuronal differentiation of NSPCs $[\underline{64}]$.

PUFAs have unique biophysical properties in membranes, regulating their interactions with proteins. They favor the occurrence of Ld membrane microdomains [29][다], which are associated with changes in protein-lipid interactions. In this context, a decline in DHA biosynthesis correlates with cognitive impairment in $A D$ patients ${ }^{[\underline{66]}]}$ and alterations to membrane lipids in neurons have been proposed as upstream events implicated in neurodegeneration, such as $A \beta$ production and tau phosphorylation $[\underline{59}]$. These lipid alterations might affect protein-lipid interactions that would activate the neurodegenerative cascade, as well as modulating neuroprotection and neuroregeneration[1] [ㄷ]]. Indeed, treatment with the PUFA 2-hydroxydocosahexaenoic acid inhibits amyloid production, tau phosphorylation, and it induces an increase in PUFAs and the recovery of cognitive scores in a mouse model of human $A D$ (5XFAD mice $\left.{ }^{[11]}\right)$.

\section{Protein-Lipid Interactions in Diabetes}

Insulin resistance has been widely associated with an altered cell membrane composition, particularly in Type-2 diabetes mellitus (T2DM). Insulin resistance is characterized by a restriction in the ability of insulin to exert its physiological functions in tissues, leading to insulin hypersecretion by the pancreas as a compensatory mechanism to maintain glucose homeostasis. Unfortunately, this hyperinsulinemia induced by insulin resistance contributes to pancreatic $\beta$-cell failure and the further development of diabetes $[\underline{68]}$. Insulin Receptor (IR) activation and its affinity for insulin depends on the cell membrane composition and structure. Decreased membrane fluidity caused by a high saturated FA content leads to less IR in the plasma membrane and reduced insulin affinity. However, the presence of PUFAs (particularly omega-3 PUFAs like DHA) increases membrane fluidity and insulin sensitivity $[\underline{69}]$.

In diabetic patients, DAG levels are chronically elevated in many peripheral tissues, leading to abnormal

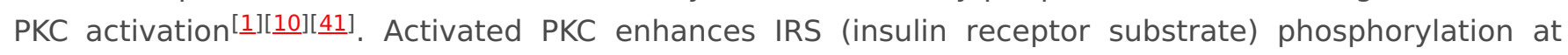
Ser/Thr residues, which inhibits a conformational change in IRS that is necessary for IR-mediated Tyr phosphorylation and insulin signaling via Phosphatidylinositol 3-kinase (PI3K) ${ }^{[70]}$. However, omega-3 PUFAs inhibit PKC to favor insulin signaling[71]. The lipid composition of the plasma membrane also influences glucose transport via GLUT. Indeed, epidemiological studies indicate that dietary changes from unsaturated towards saturated lipids inhibit the insertion of GLUT4 into the plasma membrane, thereby altering glucose uptake from the blood and insulin sensitivity [르. By contrast, experimental Chol depletion increases the density of GLUT4 receptors at the plasma membrane[그] . Interestingly, GLUT4 translocation to the plasma membrane is, in part, controlled by activation of the IR-IRS-PI3K axis which means that an increase in membrane fluidity (mediated by PUFA enrichment) in the presence of insulin may activate GLUT4 translocation to the plasma membrane[그] Finally, GLUT4 expression is under the control of PPARy, such that the presence of DHA in cell membranes and an optimal omega- 3 to omega- 6 ratio may promote GLUT4 expression[피] . Together, this evidence suggests that the membrane lipid composition acts as a switch that regulates the cell's sensitivity to insulin, whereby lipids that promote membrane fluidity like omega-3 PUFAs potentiate the insulin response and activate the enzymatic machinery for glucose uptake.

There is abundant evidence demonstrating the association between dietary fats and diabetes, which supports the use of dietary fat interventions and melitherapy as therapeutic strategies in diabetic patients. Several studies have reviewed the use of Monounsaturated Fatty Acids (MUFAs) and PUFAs in diabetes although the effect of omega-3 PUFAs in preventing insulin resistance in animals appears to be more robust $[\underline{75}]$. Increases in the unsaturation index in the cell membrane, and particularly in omega-3 PUFAs, is associated with stronger insulin sensitivity $[\underline{76]}$. In general, improved insulin sensitivity has been associated with the enrichment of omega-3 PUFAs in cell membranes, and although the exact 
mechanism mediating this effect is not yet fully understood, protein-lipid interactions probably play a relevant role in the control of glycemia[ ${ }^{[69]}$. Therefore, the biophysical properties of lipid bilayers and structural membrane dynamics may play crucial roles in diabetic patients that could influence their pathological status and its treatment.

\section{Protein-Lipid Interactions in Cardiovascular Diseases (CVDs).}

The CVDs are the leading causes of death and disability worldwide. They include heart disease, vascular diseases of the brain and other diseases of blood vessels ${ }^{[77]}$. The major risk factors for CVDs are raised blood pressure (hypertension), raised blood sugar (diabetes) and raised blood cholesterol (Chol) (hyperlipidemia), together with other conditions such as cardiac arrhythmia, congenital heart disease, rheumatic heart disease and Chagas disease (American trypanosomiasis).

Lipid molecules that alter lipid-protein interactions may have therapeutic value in CVDs. Dietary control is one of the main tools in the prevention of CVD and in therapeutic terms $\underline{\text { [7]] }} \underline{\text { 78] }}$. The benefits of the Mediterranean diet for CVDs have become generally accepted and recent studies detail the usefulness of dietary supplementation strategies based on this diet. Extra virgin olive oil or mixed nuts decrease the cases of stroke, myocardial infarction and CV mortality[굴. There are several molecular entities that affect lipid-protein interactions and that may underlie these benefits. The levels of specific fatty acids increase upon olive oil consumption and this produces an increase in the MUFAs:SFA (Saturated FA) ratio. This increase alters membrane lipid structure and membrane fluidity, favoring non-lamellar membrane structures, and affecting the position and activity of certain proteins like $G$ proteins and PKC $\left[{ }^{[80}\right]$. Both GPCRs and G proteins are sensitive to the lipid environment $[$ [ $]$ and the membraneassociation of $\mathrm{G}$ proteins and PKC is significantly impaired in hypertensive subjects. Adrenergic receptors are especially relevant for CVDs, the levels of which vary with age and they can be targeted with ß-blockers. In particular, ß-adrenergic mediated vasorelaxation and Gas coupling decreases with age and thus, melitherapy seems a plausible strategy to counteract this reduction (reviewed in [80]). The levels of lipoprotein lipase (LPL), a water-soluble enzyme responsible for hydrolyzing triglycerides in lipoproteins, and for the uptake of Chol-rich lipoproteins and of FFAs, decrease upon olive oil supplementation. This change is mediated by microRNA-410, which targets the 3'-untranslated region of the LPL gene $\underline{[81]}$.

As indicated above, hypertension is a major risk factor for CVDs which is accompanied by alterations in membrane Chol or phospholipid content, as well as in the degree of fatty acid saturation and

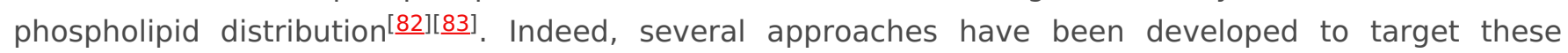
molecular alterations. For example, the MUFA 2-hydroxyoleic acid (2OHOA) is a synthetic derivative of the oleic acid (OA), inspired by the beneficial effects on hypertension of long-term high-dose OA supplementation ${ }^{[84}$ ] . The anti-hypertensive potential of 20 HOA was shown in Sprague-Dawley (S-D) and spontaneously hypertensive rats (SHRs) ${ }^{[6]}[\underline{85}]$. Sustained, time-dependent decreases in blood pressure were reported that did not affect heart rate. At the molecular level, there was more Gas in the aorta and heart membranes of S-D rats, and G $\alpha q / 11$ and $\mathrm{PKC} \alpha$ in heart membranes alone, producing increased CAMP and promoting vasodilatation. Treatment of SHRs with 2-OHOA produced a normalization of the aortic Rho kinase, suppressing the vasoconstrictor Rho kinase pathway seen in SHRs[로]

Finally, raised blood Chol is also a major risk factor for CVDs. The contribution of altered lipid profiles to the damage following stroke was proposed almost 25 years ago[ ${ }^{[6]}$. Stroke-induced energy failure is followed by FFA release from the plasma membrane of damaged cells, some of which expand ischemic damage (i.e. AA), while others exert a pro-survival effect. AA is subject to the action of cyclooxygenases (COX) and lipoxygenases (LOX), converting it into proinflammatory eicosanoids (prostaglandins, thromboxanes and leukotrienes). Accordingly, 2-hydroxy arachidonic acid (2-OAA) is a rationally designed derivative of AA known to be a competitive inhibitor of COX-1 and COX-2, and thus, it can be used in LPS-treated mice to decrease proinflammatory cytokines in serum (reviewed in [86]). When 
assessed for the treatment of stroke using S-D rats, 2-OAA treatment produced neuroprotection $\underline{\text { [87]. At }}$ the molecular level, 2-OAA decreased phospholipase A2 (PLA2) in the cell membrane with a subsequent decrease in FFA release. Therefore, the use of rationally designed lipids would seem to be a promising new stroke therapy[루].

\section{Protein-Lipid interactions in Infectious Diseases}

Bacterial membranes show important differences with respect to eukaryotic cell membranes[요, which has two relevant implications: different types of protein-lipid interactions can be found and these differences may allow the development of new therapeutic strategies to treat infectious diseases, using compounds that produce specifically effect only on prokaryotic cell membranes. Given the increased resistance of infectious microorganisms to conventional antibiotics, alternative drugs are potentially interesting to combat infections.

There are two examples supporting the relevance of protein-lipid interactions in infectious microorganisms, in which the selectivity of lipid binding to membrane protein complexes has been

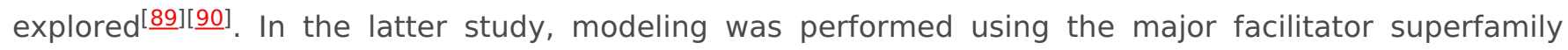
(MFS), which includes thousands of closely related secondary active and passive solute transporters, such as multidrug efflux pumps $[\underline{91}][\underline{92}]$. The MFS group includes most of the known secondary transporters, such as transporters implicated in many human pathologies, in resistance to chemotherapeutic agents in humans and in resistance to antibiotics in bacteria[ $\underline{93}$ [ [94] . Direct interactions between PE and the charge networks stabilize the inward-facing conformation, facilitating substrate release into the cytosol. It was therefore speculated that conformational regulation by specific lipidprotein interactions constitutes a widespread mechanism employed by many transporters, such as the clinically relevant solute carrier (SLC) transporters $[\underline{95]}$. These studies illustrate how lipids fine tune the structure and function of membrane proteins, through their relative abundance and the differences in their selectivity for amino acid residues ${ }^{[96]}$. Specifically, in infectious diseases this regulation influences both the interaction of the pathogenic organism with the host cell and the reaction of the immunological cells involved in the response to the pathogenic organism or condition.

\section{Summary}

Membrane lipid composition and strucutre play a crucial role in the interaction of peripheral membrane proteins with the lipid bilayer, which is mediated by the binding of these signaling proteins to specific lipid species and to supramolecular membrane structures, known as membrane microdomains. Microdomains such as caveolae, lipid rafts, liquid disordered domains, etc., act as signal propagation platforms where signaling proteins have a higher probability of physically interacting. These proteins also bear lipid or amino acid motifs that drive their interactions with specific lipid species or lipid structures. Therefore, relevant changes in membrane lipids can induce translocation of peripheral proteins from or to the plasma membrane. We have called these changes "lipid switches", as they alter the cell's proliferation, differentiation, death, etc., in response to the modulation of membrane lipids. This lipid modulation enables therapeutic interventions known as membrane-lipid therapy (MLT) or melitherapy.

\section{References}

1. Pablo Escribá; M. Sastre; J. A. García-Sevilla; Disruption of cellular signaling pathways by daunomycin through destabilization of nonlamellar membrane structures.. Proceedings of the National Academy of Sciences1995, 92, 7595-7599, 10.1073/pnas.92.16.7595.

2. Rafael Alvarez; David J. Lopez; Jesús Casas; Victoria Lladó; Mónica Higuera; Tünde Nagy; Miquel Barceló; Xavier Busquets; Pablo Escribá; G protein-membrane interactions I: Gai1 myristoyl and palmitoyl modifications in proteinlipid interactions and its implications in membrane microdomain localization. Biochimica et Biophysica Acta (BBA) Molecular and Cell Biology of Lipids 2015, 1851, 1511-1520, 10.1016/j.bbalip.2015.08.001.

3. Pablo Escribá; Antonio Ferrer-Montiel; Jose A. Ferragut; José Manuel González Ros; Role of membrane lipids in the 
interaction of daunomycin with plasma membranes from tumor cells: implications in drug-resistance phenomena. Biochemistry 1990, 29, 7275-7282, 10.1021/bi00483a017.

4. Gwendolyn Barceló-Coblijn; Maria Laura Martin; Rodrigo De Almeida; Maria Antònia Noguera-Salvà; Amaia MarcillaEtxenike; Francisca Guardiola-Serrano; Anja Lüth; Burhard Kleuser; John E. Halver; Pablo Escribá; et al. Sphingomyelin and sphingomyelin synthase (SMS) in the malignant transformation of glioma cells and in 2hydroxyoleic acid therapy.. Proceedings of the National Academy of Sciences2011, 108, 19569-74, 10.1073/pnas.1115484108.

5. Roland Seifert; Katharina Wenzel-Seifert; Constitutive activity of G-protein-coupled receptors: cause of disease and common property of wild-type receptors. Naunyn-Schmiedeberg's Archives of Pharmacology 2002, 366, 381-416, 10.1007/s00210-002-0588-0.

6. Regina Alemany; Silvia Terés; Carmela Baamonde; Mikhail Benet; Oliver Vögler; Pablo Escribá; 2-Hydroxyoleic Acid. Hypertension 2004, 43, 249-254, 10.1161/01.hyp.0000107778.85528.b5.

7. Maria A. Noguera-Salvà; Francisca Guardiola-Serrano; Maria Laura Martin; Amaia Marcilla-Etxenike; Martin O. Bergo; Xavier Busquets; Pablo Escribá; Role of the C-terminal basic amino acids and the lipid anchor of the Gy2 protein in membrane interactions and cell localization. Biochimica et Biophysica Acta (BBA) - Biomembranes 2017, 1859, 1536-1547, 10.1016/j.bbamem.2017.02.012.

8. Pablo Escribá; José M. Sánchez-Dominguez; Regina Alemany; Javier S. Perona; Valentina Ruiz-GutiÉrrez; Alteration of Lipids, G Proteins, and PKC in Cell Membranes of Elderly Hypertensives. Hypertension 2003, 41, 176-182, 10.1161/01.hyp.0000047647.72162.a8.

9. Rafael Alvarez; Jesús Casas; David J. Lopez; Maitane Ibarguren; Ariadna Suari-Rivera; Silvia Terés; Francisca Guardiola-Serrano; Alexander Lossos; Xavier Busquets; Or Kakhlon; et al.Pablo Escribá Triacylglycerol mimetics regulate membrane interactions of glycogen branching enzyme: implications for therapy. Journal of Lipid Research 2017, 58, 1598-1612, 10.1194/jlr.m075531.

10. Jordi Martínez; Oliver Vögler; Jesús Casas; Francisca Barceló; Regina Alemany; Jesús Prades; Tünde Nagy; Carmela Baamonde; Philip G. Kasprzyk; Silvia Terés; et al.Carlos SausPablo Escribá Membrane Structure Modulation, Protein Kinase C $\alpha$ Activation, and Anticancer Activity of Minerval. Molecular Pharmacology 2004, 67, 531-540, 10.1124/mol.104.000778.

11. Manuel Torres; Samantha L. Price; Maria A. Fiol-Deroque; Amaia Marcilla-Etxenike; Hasna Ahyayauch; Gwendolyn Barceló-Coblijn; Silvia Terés; Loukia Katsouri; Margarita Ordinas; David J. Lopez; et al.Maitane IbargurenFélix M. GoñiXavier BusquetsJavier VitoricaMagdalena SastrePablo Escribájavier Vitorica Ferrández Membrane lipid modifications and therapeutic effects mediated by hydroxydocosahexaenoic acid on Alzheimer's disease. Biochimica et Biophysica Acta (BBA) - Biomembranes 2014, 1838, 1680-1692, 10.1016/j. bbamem.2013.12.016.

12. Silvia Teres; Gwendolyn Barceló-Coblijn; M. Benet; R. Alvarez; R. Bressani; John E. Halver; Pablo Escribá; Oleic acid content is responsible for the reduction in blood pressure induced by olive oil. Proceedings of the National Academy of Sciences 2008, 105, 13811-13816, 10.1073/pnas.0807500105.

13. Daniel Horacio Lopez; Maria A. Fiol-Deroque; Maria A. Noguera-Salvà; Silvia Terés; Federica Campana; Stefano Piotto; J.A. Castro; Raheem J. Mohaibes; Pablo Escribá; Xavier Busquets; et al. 2-Hydroxy Arachidonic Acid: A New NonSteroidal Anti-Inflammatory Drug. PLOS ONE 2013, 8, e72052, 10.1371/journal.pone.0072052.

14. Pablo Escribá; Membrane-lipid therapy: a new approach in molecular medicine.Trends in Molecular Medicine 2006, 12, 34-43, 10.1016/j.molmed.2005.11.004.

15. Oliver Vögler; Jesús Casas; Gudrun Borchert; Danita Capó; Tünde Nagy; Gabriel Martorell; Pablo Escribá; The GßY Dimer Drives the Interaction of Heterotrimeric GiProteins with Nonlamellar Membrane Structures. Journal of Biological Chemistry 2004, 279, 36540-36545, 10.1074/jbc.m402061200.

16. Pablo Escribá; Andrés Ozaita; Catalina Ribas; Antoni Miralles; Elfrieda Fodor; Tibor Farkas; Jesús A. García-Sevilla; Role of lipid polymorphism in G protein-membrane interactions: Nonlamellar-prone phospholipids and peripheral protein binding to membranes. Proceedings of the National Academy of Sciences1997, 94, 11375-11380, 10.1073/pnas.94.21.11375.

17. John R. Silvius; Cholesterol modulation of lipid intermixing in phospholipid and glycosphingolipid mixtures. Evaluation using fluorescent lipid probes and brominated lipid quenchers. Biochemistry 1992, 31, 3398-3408, 10.1021/bi00128a014.

18. Susan Mabrey; Pedro L. Mateo; Julian M. Sturtevant; High-sensitivity scanning calorimetric study of mixtures of cholesterol with dimyristoyl- and dipalmitoylphosphatidylcholines. Biochemistry 1978, 17, 2464-2468, 10.1021/bi00605a034.

19. Pablo Escribá; Membrane-lipid therapy: A historical perspective of membrane-targeted therapies — From lipid bilayer structure to the pathophysiological regulation of cells. Biochimica et Biophysica Acta (BBA) - Biomembranes2017, 1859, 1493-1506, 10.1016/j.bbamem.2017.05.017.

20. Luis M. García-Segura; Jose A. Ferragut; Antonio Ferrer-Montiel; Pablo Escribá; José Manuel González Ros; Ultrastructural alterations in plasma membranes from drug-resistant P388 murine leukemia cells. Biochimica et Biophysica Acta (BBA) - Biomembranes 1990, 1029, 191-195, 10.1016/0005-2736(90)90454-v. 
21. Pablo Escribá; Xavier Busquets; Jin-Ichi Inokuchi; Gábor Balogh; Zsolt Török; Ibolya Horvàth; John L. Harwood; László Vígh; Membrane lipid therapy: Modulation of the cell membrane composition and structure as a molecular base for drug discovery and new disease treatment. Progress in Lipid Research 2015, 59, 38-53, 10.1016/j.plipres.2015.04.003.

22. Félix M. Goñi; “Rafts”: A nickname for putative transient nanodomains. Chemistry and Physics of Lipids 2019, 218, 34-39, 10.1016/j.chemphyslip.2018.11.006.

23. T. Triton; G Yee; The anticancer agent adriamycin can be actively cytotoxic without entering cells Science $\mathbf{1 9 8 2}$, 217, 248-250, 10.1126/science.7089561.

24. Chieko Mineo; Guy L. James; Eric J. Smart; Richard G. W. Anderson; Localization of Epidermal Growth Factorstimulated Ras/Raf-1 Interaction to Caveolae Membrane. Journal of Biological Chemistry 1996, 271, 11930-11935, 10.1074/jbc.271.20.11930.

25. Ok-Ho Shin; Exocytosis and Synaptic Vesicle Function. Comprehensive Physiology 2014, 4, 149-175, 10.1002/cphy.c130021.

26. P L Yeagle; Lipid regulation of cell membrane structure and function..The FASEB Journal 1989, 3, 1833-1842, 10.1096/fasebj.3.7.2469614.

27. F M Goñi; A Alonso; Structure and functional properties of diacylglycerols in membranes..Progress in Lipid Research 1999, 38, 1-48, 10.1016/S0163-7827(98)00021-6.

28. Siewert J. Marrink; Alan E. Mark; Molecular View of Hexagonal Phase Formation in Phospholipid Membranes. Biophysical Journal 2004, 87, 3894-3900, 10.1529/biophysj.104.048710.

29. Maitane Ibarguren; David J. Lopez; José Antonio Encinar; José Manuel González Ros; Xavier Busquets; Pablo Escribá; Partitioning of liquid-ordered/liquid-disordered membrane microdomains induced by the fluidifying effect of 2hydroxylated fatty acid derivatives. Biochimica et Biophysica Acta (BBA) - Biomembranes2013, 1828, 2553-2563, 10.1016/j.bbamem.2013.06.014.

30. Lin Feng; Wu-Xiang Liao; Quan Luo; Hong-Hai Zhang; Wen Wang; Jing Zheng; Ng-Bao Chen; Caveolin-1 orchestrates fibroblast growth factor 2 signaling control of angiogenesis in placental artery endothelial cell caveolae. Journal of Cellular Physiology 2012, 227, 2480-91, 10.1002/jcp.22984.

31. Peter J. Quinn; Dennis Chapman; Alec D. Keith; The Dynamics Of Membrane Structure.Critical Reviews in Biochemistry 1980, 8, 1-117, 10.3109/10409238009105466.

32. Doralicia Casares; Pablo Escribá; Catalina Ana Rosselló; Membrane Lipid Composition: Effect on Membrane and Organelle Structure, Function and Compartmentalization and Therapeutic Avenues.. International Journal of Molecular Sciences 2019, 20, 2167, 10.3390/ijms20092167.

33. Heike Grassmé; Brian Henry; Regan Ziobro; Katrin Anne Becker; Joachim Riethmüller; Aaron Gardner; Aaron P. Seitz; Joerg Steinmann; Stephan Lang; Christopher L. Ward; et al.Edward H. SchuchmanCharles C. CaldwellMarkus KamlerMichael J. EdwardsMalcolm BrodlieErich Gulbins $\beta 1$-Integrin Accumulates in Cystic Fibrosis Luminal Airway Epithelial Membranes and Decreases Sphingosine, Promoting Bacterial Infections.. Cell Host \& Microbe 2017, 21, 707-718.e8, 10.1016/j.chom.2017.05.001.

34. Lasantha Gunasekara; Mustafa Al-Saiedy; Francis H.Y. Green; Ryan Pratt; Candice Bjornson; Ailian Yang; W. Michael Schoel; Ian Mitchell; Mary Brindle; Mark Montgomery; et al.Elizabeth KeysJohn DennisGrishma ShresthaMatthias Amrein Pulmonary surfactant dysfunction in pediatric cystic fibrosis: Mechanisms and reversal with a lipidsequestering drug. Journal of Cystic Fibrosis 2017, 16, 565-572, 10.1016/j.jcf.2017.04.015.

35. S. D. Freedman; M. H. Katz; E. M. Parker; M. Laposata; M. Y. Urman; J. G. Alvarez; A membrane lipid imbalance plays a role in the phenotypic expression of cystic fibrosis in cftr-/- mice. Proceedings of the National Academy of Sciences 1999, 96, 13995-14000, 10.1073/pnas.96.24.13995.

36. Joseph O. Ojo; Moustafa Algamal; Paige Leary; Laila Abdullah; Benoit Mouzon; James E. Evans; Michael Mullan; Fiona Crawford; Disruption in Brain Phospholipid Content in a Humanized Tau Transgenic Model Following Repetitive Mild Traumatic Brain Injury. Frontiers in Neuroscience 2018, 12, 1-18, 10.3389/fnins.2018.00893.

37. Pedro Geraldes; George L. King; Activation of protein kinase C isoforms and its impact on diabetic complications.. Circulation Research 2010, 106, 1319-31, 10.1161/CIRCRESAHA.110.217117.

38. Kuo-Ping Huang; The mechanism of protein kinase C activation.Trends in Neurosciences 1989, 12, 425-432, 10.1016/0166-2236(89)90091-x.

39. Elena Oancea; Tobias Meyer; Protein kinase $C$ as a molecular machine for decoding calcium and diacylglycerol signals.. Cell 1998, 95, 307-318, 10.1016/s0092-8674(00)81763-8.

40. Stéphane Yerly; Heidrun Ding; Sebastien Tauzin; Bettina Borisch; Gerhild Van Echten-Deckert; Daniel C. Hoessli; The sphingolipid-rich rafts of ALK+ lymphomas downregulate the Lyn-Cbp/PAG signalosome. European Journal of Haematology 2010, 85, 93-98, 10.1111/j.1600-0609.2010.01492.x.

41. Silvia Terés; Victoria Lladó; Mónica Higuera; Gwendolyn Barceló-Coblijn; Maria Laura Martin; Maria Antònia NogueraSalvà; Amaia Marcilla-Etxenike; Jose Manuel García-Verdugo; Mario Soriano-Navarro; Carlos Saus; et al.Ulises.Gomez- PinedoXavier BusquetsPablo Escribá 2-Hydroxyoleate, a nontoxic membrane binding anticancer drug, induces glioma cell differentiation and autophagy. Proceedings of the National Academy of Sciences2012, 
109, 8489-8494, 10.1073/pnas.1118349109.

42. Sara Hernando; Catalina Requejo; Enara Herran; Jose Angel Ruiz-Ortega; Teresa Morera-Herreras; Jose-Vicente Lafuente; Luisa Ugedo; Eusebio Gainza; Jose Luis Pedraz; Manoli Igartua; et al.Ardeshir Bayat Beneficial effects of n-3 polyunsaturated fatty acids administration in a partial lesion model of Parkinson's disease: The role of glia and NRf2 regulation. Neurobiology of Disease 2019, 121, 252-262, 10.1016/j.nbd.2018.10.001.

43. Ian A Prior; Keir Lewis; Carla Mattos; A comprehensive survey of Ras mutations in cancer..Cancer Research 2012, 72, 2457-67, 10.1158/0008-5472.CAN-11-2612.

44. Walaa E Kattan; Wei Chen; Xiaoping Ma; Tien Hung Lan; Dharini Van Der Hoeven; Ransome Van Der Hoeven; John Hancock; Targeting plasma membrane phosphatidylserine content to inhibit oncogenic KRAS function.. Life Science Alliance 2019, 2, e201900431, 10.26508/lsa.201900431.

45. Boris Bleijlevens; Mariëlle J. Van Breemen; Wilma E. Donker-Koopman; Chris De Koster; Johannes M.F.G Aerts; Detection of mutant protein in complex biological samples: Glucocerebrosidase mutations in Gaucher's disease. Analytical Biochemistry 2008, 372, 52-61, 10.1016/j.ab.2007.09.033.

46. Rachel H. Giles; Johan H. Van Es; Hans Clevers; Caught up in a Wnt storm: Wnt signaling in cancerBiochimica et Biophysica Acta (BBA) - Reviews on Cancer 2003, 1653, 1-24, 10.1016/s0304-419x(03)00005-2.

47. Baoen Chen; Yang Sun; Jixiao Niu; Gopala K. Jarugumilli; Xu Wu; Protein Lipidation in Cell Signaling and Diseases: Function, Regulation, and Therapeutic Opportunities. Cell Chemical Biology 2018, 25, 817-831, 10.1016/j.chembiol.2018.05.003.

48. Abigail K. Suwala; Allison Hanaford; Ulf D. Kahlert; Jaroslaw Maciaczyk; Clipping the Wings of Glioblastoma: Modulation of WNT as a Novel Therapeutic Strategy. Journal of Neuropathology \& Experimental Neurology 2016, 75, 388-396, 10.1093/jnen/nlw013.

49. Jon C. Aster; Warren S. Pear; Stephen C. Blacklow; The Varied Roles of Notch in Cancer.Annual Review of Pathology: Mechanisms of Disease 2017, 12, 245-275, 10.1146/annurev-pathol-052016-100127.

50. Ann Hanna; Lalita A. Shevde; Hedgehog signaling: modulation of cancer properies and tumor mircroenvironment.. Molecular Cancer 2016, 15, 24, 10.1186/s12943-016-0509-3.

51. Sara Sigismund; Daniele Avanzato; Letizia Lanzetti; Emerging functions of the EGFR in cancer.Molecular Oncology 2018, 12, 3-20, 10.1002/1878-0261.12155.

52. Lenka Doubravská; Michaela Krausova; Dietmar Gradl; Martina Vojtechova; Lucie Tumova; Jan Lukas; Tomas Valenta; Vendula Pospíchalová; Bohumil Fafilek; Jiri Plachy; et al.Ondrej SebestaVladimir Korinek Fatty acid modification of Wnt1 and Wnt3a at serine is prerequisite for lipidation at cysteine and is essential for Wnt signalling. Cellular Signalling 2011, 23, 837-848, 10.1016/j.cellsig.2011.01.007.

53. Victoria Lladó; David J. Lopez; Maitane Ibarguren; Maria Alonso; Joan B. Soriano; Pablo Escribá; Xavier Busquets; Regulation of the cancer cell membrane lipid composition by NaCHOleate. Biochimica et Biophysica Acta (BBA) Biomembranes 2014, 1838, 1619-1627, 10.1016/j.bbamem.2014.01.027.

54. Marlen Knobloch; The Role of Lipid Metabolism for Neural Stem Cell Regulation.Brain Plasticity 2017, 3, 61-71, 10.3233/bpl-160035.

55. Michael J. Weiser; Christopher M. Butt; M. Hasan Mohajeri; Docosahexaenoic Acid and Cognition throughout the Lifespan. Nutrients 2016, 8, 99, 10.3390/nu8020099.

56. Motoko Maekawa; Noriko Takashima; Miho Matsumata; Shiro Ikegami; Masanori Kontani; Yoshinobu Hara; Hiroshi Kawashima; Yuji Owada; Yoshinobu Kiso; Takeo Yoshikawa; et al.Kaoru InokuchiNoriko Osumi Arachidonic Acid Drives Postnatal Neurogenesis and Elicits a Beneficial Effect on Prepulse Inhibition, a Biological Trait of Psychiatric IIInesses. PLOS ONE 2009, 4, e5085, 10.1371/journal. pone.0005085.

57. Nobuyuki Sakayori; Motoko Maekawa; Keiko Numayama-Tsuruta; Takashi Katura; Takahiro Moriya; Noriko Osumi; Distinctive effects of arachidonic acid and docosahexaenoic acid on neural stem/progenitor cells. Neuroscience Research 2011, 71, e228, 10.1016/j.neures.2011.07.995.

58. Nobuyuki Sakayori; Takako Kikkawa; Hisanori Tokuda; Emiko Kiryu; Kaichi Yoshizaki; Hiroshi Kawashima; Tetsuya Yamada; Hiroyuki Arai; Jing X. Kang; Hideki Katagiri; et al.Hiroshi ShibataSheila M. InnisMakoto AritaNoriko Osumi Maternal dietary imbalance between omega- 6 and omega-3 polyunsaturated fatty acids impairs neocortical development via epoxy metabolites. STEM CELLS 2016, 34, 470-482, 10.1002/stem.2246.

59. Manuel Torres; Xavier Busquets; Pablo Escribá; Brain Lipids in the Pathophysiology and Treatment of Alzheimer's Disease. Update on Dementia; In Tech (Rijeka, Croatia) 2016, Chapter 7, 127-167, 10.5772/64757.

60. Bénédicte Langelier; Alain Linard; Christian Bordat; Monique Lavialle; Christine Heberden; Long chainpolyunsaturated fatty acids modulate membrane phospholipid composition and protein localization in lipid rafts of neural stem cell cultures. Journal of Cellular Biochemistry 2010, 110, 1356-1364, 10.1002/jcb.22652.

61. Rong-Zong Liu; Raja Mita; Michaël Beaulieu; Zhihua Gao; Roseline Godbout; Fatty acid binding proteins in brain development and disease. The International Journal of Developmental Biology 2010, 54, 1229-1239, 10.1387/ijdb.092976rl.

62. Miho Matsumata; Hitoshi Inada; Noriko Osumi; Fatty acid binding proteins and the nervous system: Their impact on mental conditions. Neuroscience Research 2016, 102, 47-55, 10.1016/j.neures.2014.08.012. 
63. Giuseppe Esposito; Caterina Scuderi; Marta Valenza; Giuseppina Ines Togna; Valentina Latina; Daniele De Filippis; Mariateresa Cipriano; Maria Carratù; Teresa luvone; Luca Steardo; et al. Cannabidiol Reduces A $\beta$-Induced Neuroinflammation and Promotes Hippocampal Neurogenesis through PPARy Involvement. PLOS ONE 2011, 6, e28668, 10.1371/journal.pone.0028668.

64. Dexuan Ma; Minmin Zhang; Christian P. Larsen; Feng Xu; Wei Hua; Tetsumori Yamashima; Ying Mao; Liangfu Zhou; DHA promotes the neuronal differentiation of rat neural stem cells transfected with GPR40 gene. Brain Research 2010, 1330, 1-8, 10.1016/j.brainres.2010.03.002.

65. Maitane Ibarguren; David J. Lopez; Pablo Escribá; The effect of natural and synthetic fatty acids on membrane structure, microdomain organization, cellular functions and human health. Biochimica et Biophysica Acta (BBA) Biomembranes 2014, 1838, 1518-1528, 10.1016/j.bbamem.2013.12.021.

66. Giuseppe Astarita; Kwang-Mook Jung; Nicole C. Berchtold; Vinh Q. Nguyen; Daniel L. Gillen; Elizabeth Head; Carl W. Cotman; Daniele Piomelli; Deficient Liver Biosynthesis of Docosahexaenoic Acid Correlates with Cognitive Impairment in Alzheimer's Disease. PLOS ONE 2010, 5, e12538, 10.1371/journal. pone.0012538.

67. Maria A. Fiol-Deroque; Raquel Gutiérrez-Lanza; Silvia Terés; Manuel Torres; Pere Barceló; Rubén V. Rial; Alexei Verkhratsky; Pablo Escribá; Xavier Busquets; José Julio Rodíguez Arellano; et al. Cognitive recovery and restoration of cell proliferation in the dentate gyrus in the 5XFAD transgenic mice model of Alzheimer's disease following 2hydroxy-DHA treatment. Biogerontology 2013, 14, 763-775, 10.1007/s10522-013-9461-4.

68. Kitt Falk Petersen; Gerald I. Shulman; Etiology of insulin resistance..The American Journal of Medicine 2006, 119, S10-6, 10.1016/j.amjmed.2006.01.009.

69. Undurti N. Das; $A$ defect in the activity of $\Delta 6$ and $\Delta 5$ desaturases may be a factor predisposing to the development of insulin resistance syndrome. Prostaglandins, Leukotrienes and Essential Fatty Acids 2005, 72, 343-350, 10.1016/j.plefa.2005.01.002.

70. Ellen Dirkx; Robert W. Schwenk; Jan F.C. Glatz; Joost J.F.P. Luiken; Guillaume J.J.M. Van Eys; High fat diet induced diabetic cardiomyopathy. Prostaglandins, Leukotrienes and Essential Fatty Acids 2011, 85, 219-225, 10.1016/j.plefa.2011.04.018.

71. Marianne Haag; Nola G Dippenaar; Dietary fats, fatty acids and insulin resistance: short review of a multifaceted connection.. Medical Science Monitor 2005, 11, RA359-RA367, PMID: 16319806.

72. Rob N. M. Weijers; Lipid composition of cell membranes and its relevance in type 2 diabetes mellitus. Current Diabetes Reviews 2012, 8, 390-400, 10.2174/157339912802083531.

73. Satoshi Shigematsu; Robert T. Watson; Ahmir H. Khan; Jeffrey E. Pessin; Tim J. Craig; Richard J. Fisher; Leonora F. Ciufo; Gareth J. O. Evans; Jeff W. Barclay; Alan Morgan; et al.Robert Burgoyne The Adipocyte Plasma Membrane Caveolin Functional/Structural Organization Is Necessary for the Efficient Endocytosis of GLUT4. Journal of Biological Chemistry 2003, 278, 10683-10690, 10.1074/jbc.m208563200.

74. Alan Dresner; Didier Laurent; Melissa Marcucci; Margaret E. Griffin; Sylvie Dufour; Gary W. Cline; Lori A. Slezak; Dana K. Andersen; Ripudaman S. Hundal; Uglas L. Rothman; et al.Kitt Falk PetersenGerald I. Shulman Effects of free fatty acids on glucose transport and IRS-1-associated phosphatidylinositol 3-kinase activity. Journal of Clinical Investigation 1999, 103, 253-259, 10.1172/jci5001.

75. Trevor Mori; Dietary n-3 PUFA and CVD: a review of the evidence.Proceedings of the Nutrition Society 2013, 73, 5764, 10.1017/s0029665113003583.

76. Chao-Wei Huang; Yi-Shan Chien; Yu-Jen Chen; Kolapo M. Ajuwon; Harry John Mersmann; Shih-Torng Ding; Role of n-3 Polyunsaturated Fatty Acids in Ameliorating the Obesity-Induced Metabolic Syndrome in Animal Models and Humans. International Journal of Molecular Sciences 2016, 17, 1689, 10.3390/ijms17101689.

77. Shanthi Mendis; P. Nordet; J.E. Fernández-Britto; N. Sternby; Atherosclerosis in children and young adults: An overview of the World Health Organization and International Society and Federation of Cardiology study on Pathobiological Determinants of Atherosclerosis in Youth study (1985-1995). Prevention and Control 2005, 1, 3-15, 10.1016/j.precon.2005.02.010.

78. Marta Guasch-Ferré; Frank B. Hu; Miguel Ángel Martínez-González; Montserrat Fitó; Monica Bulló; Ramon Estruch; Emilio Ros; Dolores Corella; Javier Recondo; Enrique Gómez-Gracia; et al.Miquel FiolJosé LapetraLluís SerraMajemMiguel-Angel MunozXavier PintóRosa M. Lamuela-RaventósJosep BasoraPilar Buil-Cosialesjose V. SorlíValentina Ruíz-GutierrezAlfredo MartínezJordi Salas-Salvadó Olive oil intake and risk of cardiovascular disease and mortality in the PREDIMED Study. BMC Medicine 2014, 12, 78, 10.1186/1741-7015-12-78.

79. Miguel Ángel Martínez-González; Estefanía Toledo; Fernando Arós; Miquel Fiol; Dolores Corella; Jordi Salas-Salvadó; Emilio Ros; Maria I. Covas; Joaquín Fernández-Crehuet; José Lapetra; et al.Miguel-Angel MunozMontserrat FitóLluís Serra-MajemXavier PintóRosa M. Lamuela-RaventósJose V. SorlíNancy BabioPilar Buil-CosialesValentina RuizGutierrezRamón EstruchAlvaro Alonsofor the PREDIMED Investigators* Extravirgin Olive Oil Consumption Reduces Risk of Atrial Fibrillation. Circulation 2014, 130, 18-26, 10.1161/circulationaha.113.006921.

80. Regina Alemany; Javier S. Perona; José M. Sánchez-Dominguez; Emilio Montero; Julio Cañizares; Ricardo Bressani; Pablo Escribá; Valentina Ruíz-Gutierrez; G protein-coupled receptor systems and their lipid environment in health disorders during aging. Biochimica et Biophysica Acta (BBA) - Biomembranes2007, 1768, 964-975, 
10.1016/j.bbamem.2006.09.024.

81. Dolores Corella; Jose V. Sorlí; Ramón Estruch; Oscar Coltell; Carolina Ortega-Azorín; Olga Portoles; Miguel Ángel Martínez-González; Monica Bulló; Montserrat Fitó; Fernando Arós; et al.José LapetraE. M. AsensioGuillermo T. SaezLluís Serra-MajemCarlos Muñoz-BravoValentina Ruiz-GutiérrezMiquel FiolE. VinyolesXavier PintóKris RichardsonEmilio RosJosé María Ordovas MicroRNA-410 regulated lipoprotein lipase variant rs13702 is associated with stroke incidence and modulated by diet in the randomized controlled PREDIMED trial. The American Journal of Clinical Nutrition 2014, 100, 719-731, 10.3945/ajcn.113.076992.

82. J Villar; C Montilla; O Muñiz-Grijalvo; F G Muriana; P Stiefel; V Ruiz-Gutiérrez; J Carneado; Erythrocyte Na(+)-Li+ countertransport in essential hypertension: correlation with membrane lipids levels.. J. Hypertens. 1996, 14, 969973, PMID: 8884551.

83. Carla Russo; Oliviero Olivieri; Domenico Girelli; Patrizia Guarini; Roberta Pasqualini; Margherita Azzini; Roberto Corrocher; Increased membrane ratios of metabolite to precursor fatty acid in essential hypertension.. Hypertension 1997, 29, 1058-1063, 10.1161/01.hyp.29.4.1058.

84. Siegfried Heyden; Polyunsaturated and Monounsaturated Fatty Acids in the Diet to Prevent Coronary Heart Disease via Cholesterol Reduction. Annals of Nutrition and Metabolism 1994, 38, 117-122, 10.1159/000177801.

85. Regina Alemany; Oliver Vögler; Silvia Terés; Carolina Egea; Carmela Baamonde; Francisca Barceló; Carlos Delgado; Karl H. Jakobs; Pablo Escribá; Antihypertensive action of 2-hydroxyoleic acid in SHRs via modulation of the protein kinase A pathway and Rho kinase. Journal of Lipid Research 2006, 47, 1762-1770, 10.1194/jlr.m500520-jlr200.

86. Irene Fernández Ugidos; Diego Perez Rodriguez; Arsenio Fernández-López; A role for lipids as agents to alleviate stroke damage: the neuroprotective effect of 2-hydroxy arachidonic acid. Neural Regeneration Research 2017, 12, 1273-1275, 10.4103/1673-5374.213545.

87. Irene Fernández Ugidos; María Santos Galdiano; Diego Perez Rodriguez; B. Anuncibay-Soto; Enrique Font Belmonte; David J. Lopez; Maitane Ibarguren; Xavier Busquets; Arsenio Fernández-López; Neuroprotective effect of 2-hydroxy arachidonic acid in a rat model of transient middle cerebral artery occlusion. Biochimica et Biophysica Acta (BBA) Biomembranes 2017, 1859, 1648-1656, 10.1016/j.bbamem.2017.03.009.

88. Richard M. Epand; Raquel F. Epand; Domains in bacterial membranes and the action of antimicrobial agents. Molecular BioSystems 2009, 5, 580, 10.1039/b900278m.

89. Arthur Laganowsky; Eamonn Reading; Timothy M. Allison; Martin B Ulmschneider; Matteo T Degiacomi; Andrew J. Baldwin; Carol V. Robinson; Membrane proteins bind lipids selectively to modulate their structure and function. Nature 2014, 510, 172-175, 10.1038/nature13419.

90. Chloe Martens; Mrinal Shekhar; Antoni J. Borysik; Andy M. Lau; Eamonn Reading; Emad Tajkhorshid; Paula Jane Booth; Argyris Politis; Direct protein-lipid interactions shape the conformational landscape of secondary transporters. Nature Communications 2018, 9, 4151, 10.1038/s41467-018-06704-1.

91. Peter J.F. Henderson; Sugar transport proteins. Current Opinion in Structural Biology 1991, 1, 590-601, 10.1016/s0959-440x(05)80082-x.

92. Stephanie S. Pao; Ian T. Paulsen; Milton H. Saier; Major Facilitator Superfamily.Microbiology and Molecular Biology Reviews 1998, 62, 1-34, 10.1128/mmbr.62.1.1-34.1998.

93. Claire Colas; Peter Man-Un Ung; Avner Schlessinger; SLC transporters: structure, function, and drug discovery. MedChemComm 2016, 7, 1069-1081, 10.1039/c6md00005c.

94. S Kumar; G He; P Kakarla; U Shrestha; R Kc; I Ranaweera; Tm Willmon; Sr Barr; Aj Hernandez; Mf Varela; et al. Bacterial Multidrug Efflux Pumps of the Major Facilitator Superfamily as Targets for Modulation. Infectious Disorders Drug Targets 2016, 16, 1-1, 10.2174/1871526516666160407113848.

95. Lawrence Lin; Sook Wah Yee; Richard B. Kim; Kathleen M. Giacomini; SLC transporters as therapeutic targets: emerging opportunities. Nature Reviews Drug Discovery 2015, 14, 543-560, 10.1038/nrd4626.

96. Nald M. Engelman; Membranes are more mosaic than fluid. Nature 2005, 438, 578-580, 10.1038/nature04394.

\section{Keywords}

melitherapy; lipid switch; protein-membrane interactions; peripheral amphitropic membrane proteins

(C) 2020 by the author(s). Distribute under a Creative Commans CC BY license 